\title{
More Information, Less Understanding and the Need to Educate Consumers and Allied Health Professionals to Critically Evaluate Health Related Mass Communications
}

\section{Corey H Basch ${ }^{1 *}$ and Joanna Hayden ${ }^{2}$}

${ }^{1}$ Assistant Professor, Department of Public Health, William Paterson University, Wayne, New Jersey, USA

${ }^{2}$ Professor, Department of Public Health, Director, School Nurse Certification, William Paterson University, Wayne, New Jersey, USA

An overwhelming amount of health related information is communicated in the media on a daily basis, and media influence is one of the main social determinants of health. Communications about health issues are sometimes fraught with "legitimate" conflicting views, which results in confusion for consumers. There are also different messages communicated by different kinds of communications, articles, editorials, or advertising, and by different sources of information. It is, therefore, not surprising that consumers may frequently be confused about important issues such as what foods to eat, how to lose weight or stop smoking, what medicines to take, what sources of information to trust, among many other health matters. Social media such as twitter and Facebook have become other important media sources that consumers rely on for health information, and these sources may be more likely to convey opinions and experiences versus knowledge supported by science. Public health professionals now also pervasively depend on the media as a way to communicate with consumers and social marketing campaigns involving mass communications that address various topics are common. To the extent that these messages have not been evaluated and conveyed in a way that is comprehended as intended this too can add to consumers' confusion.

Health professionals may also turn to the popular media to garner information about current health issues and use this information to inform their recommendations to consumers. Without the ability to critically evaluate the information conveyed, these professionals may be hindered in their efforts to help consumers make informed decisions. Unless health professionals are cognizant of the extent to which misinformation abounds in popular media outlets, the very people who are supposed to be the experts may be at risk for perpetuating misinformation. To address this, degree programs preparing health professionals at all levels can integrate analysis of health information conveyed through mass communications into curricula.

For example, students can locate an article in a popular media outlet (newspaper, for example, either online or print version) and then retrieve the original journal article upon which the news article is reporting. This exercise does at least two things: it brings to light the possibility that the information in the news article is incorrect, incomplete, or inconsistent with the original report, and it requires the skill to locate an original article, which always contains much more detailed information. To maximize the value of such teaching/learning activities, students must learn how to critically evaluate the original research reports, which is not always straightforward.

The cost of health care is consuming an increasing proportion of the US gross national product and placing an increasing burden on the US economy. One strategy to address this is to improve the health literacy of consumers. Since so many causes of morbidity and mortality in the US are influenced by decisions under the volition of consumers, the importance of what information is conveyed, how it is conveyed, and how these communications influence consumers' decisions has become increasingly important. At the same time, health profession preparation programs and developers of health communications have a moral and ethical responsibility to ensure that the public receives accurate health information.

*Corresponding author: Corey Hannah Basch, Assistant Professor, Department of Public Health, William Paterson University Wing 150, Wayne, NJ 07470, Tel: 973720-2603; E-mail: baschc@wpunj.edu

Received January 09, 2014; Accepted January 10, 2010 ; Published January 11 2014

Citation: Basch CH, Hayden J (2014) More Information, Less Understanding and the Need to Educate Consumers and Allied Health Professionals to Critically Evaluate Health Related Mass Communications. J Mass Communicat Journalism 4: e152. doi:10.4172/2165-7912.1000e152

Copyright: $\odot 2014$ Basch CH, et al. This is an open-access article distributed under the terms of the Creative Commons Attribution License, which permits unrestricted use, distribution, and reproduction in any medium, provided the original author and source are credited. 